\title{
Correction to: A Numerical Study for the Effect of Ski Vibration on Friction
}

\author{
Yunhyoung Nam ${ }^{1} \cdot$ Jinsu Gim ${ }^{2} \cdot$ Taejoon Jeong ${ }^{2} \cdot$ Byungohk Rhee $^{2} \cdot$ Do-Nyun Kim ${ }^{1,3}$
}

Published online: 3 May 2019

(c) Korean Multi-Scale Mechanics (KMSM) 2019

\section{Correction to: Multiscale Science and Engineering https://doi.org/10.1007/s42493-018-00005-x}

The original version of this article unfortunately contained a mistake. Jinsu Gim, Taejoon Jeong and Byungohk Rhee were not listed among the authors.

According to the corresponding author, the paper presents a numerical study on how the vibration characteristics of skis affect the friction with the ice track during sliding. It was investigated by performing a comprehensive set of numerical simulations based on the computational model for skis. Unfortunately, it was only found out after publication of the article that the computational model built by the first author was constructed to represent a real ski whose geometry and stiffness were measured experimentally by the newly added authors. While the analysis is purely computational, the experimental measurements contribute to the paper because they were used to construct a ski model in this study and they can be also useful for the readers interested in building a different ski model. Therefore, it was considered necessary to give co-authorship to the new authors. All (original and new) authors agree on it. The newly added authors were accidentally omitted in the original manuscript due to lack of recognition of their contribution. More detailed author contributions are provided below:

The original article can be found online at https://doi.org/10.1007/ s42493-018-00005-x.

Do-Nyun Kim

dnkim@snu.ac.kr

1 Department of Mechanical and Aerospace Engineering, Seoul National University, 1 Gwanak-ro, Gwanak-gu, Seoul 08826, Korea

2 Department of Mechanical Engineering, Ajou University, Suwon, Republic of Korea

3 Institute of Advanced Machines and Design, Seoul National University, 1 Gwanak-ro, Gwanak-gu, Seoul 08826, Korea
Author Contributions YN and DK conceived the idea, designed the research direction, and developed the numerical analysis procedure to analyze the mechanical properties of skis and predict the effect of ski vibration on friction. YN and DK revised the theoretical model for ice friction. JG, TJ, and BR developed the experimental methods for ski measurement. JG and TJ performed the experiments and measured the geometry and properties of skis. BR directed the experiment and checked the measured values. YN constructed the computational model for skis and performed all the simulations to estimate the stiffness and the vibration characteristics of skis, and to study their effect on the friction between the icy track and the ski plate.

The original article has been corrected.

Publisher's Note Springer Nature remains neutral with regard to jurisdictional claims in published maps and institutional affiliations. 